\title{
An Open Study to Evaluate Effectiveness and Tolerability of a Nail Oil Composed of Vitamin E and Essential Oils in Mild to Moderate Distal Subungual Onychomycosis
}

\author{
Aurora Alessandrini Michela Starace Francesca Bruni Bianca Maria Piraccini \\ Department of Experimental, Diagnostic and Specialty Medicine, Division of Dermatology, University of Bologna, \\ Bologna, Italy
}

\author{
Keywords \\ Onychomycosis · Topical therapy · Tocopherol acetate · Tea \\ tree oil · Oregano oil · Lime oil
}

\begin{abstract}
Background: Onychomycosis is the most common nail disease and can affect both fingernails and toenails. When possible, topical treatment is generally preferred both by patients and physicians because it is associated with lower risk of systemic side effects and drug interactions than oral antifungals, avoiding laboratory monitoring. Objective: The aim of our study was to evaluate the efficacy, tolerability, and patient's compliance of a new topical antifungal containing vitamin $\mathrm{E}$ and essential oils of lime, oregano, and tea tree. Patients and Methods: We enrolled 20 patients with mildmoderate distal subungual onychomycosis due to dermatophytes or non-dermatophyte molds. The product was applied once daily on the periungual tissues and on the nail plate for 6 months. Follow-up without therapy continued for another 6 months in order to evaluate the product's effect maintenance. Periodic evaluation of treatment efficacy was performed by standardized photography and mycological examination ( $\mathrm{KOH}+$ culture) of the target nail at baseline (T0), after 3 months (T1) and 6 months (T2) of therapy, and
\end{abstract}

after 6 months of follow-up (T3). Results: At the end of the 12-month study, the majority of patients achieved a complete cure of onychomycosis (78.5\%). All patients were very satisfied by the treatment. No side effects were recorded. Conclusions: The results of our study indicate that this new topical antifungal containing vitamin $\mathrm{E}$ and essential oils of lime, oregano, and tea tree is an effective and safe option for topical therapy of onychomycosis. This topical antifungal nail oil restructures the nail appearance, improving patient's adherence to therapy and reducing the risk of relapses, maintaining results over time.

(c) 2019 The Author(s)

Published by S. Karger AG, Basel

\section{Introduction}

Onychomycosis is the most common nail infective disorder and it is responsible for about $50 \%$ of all consultations for nail disorders. Onychomycosis has been reported as a gender- and age-related disease, as it is more prevalent in males and its prevalence increases with age in both genders [1]. Predisposing factors include mainly systemic diseases, i.e., diabetes mellitus, peripheral arterial disease, immunosuppression.

Aurora Alessandrini

Department of Experimental, Diagnostic and Specialty Medicine Division of Dermatology, University of Bologna

Via Massarenti, 1, IT-40138 Bologna (Italy)

E-Mail aurora.alessandrini3@unibo.it

\section{(c) 2019 The Author(s) Karge}

Published by S. Karger AG, Basel Open access

This article is licensed under the Creative Commons AttributionNonCommercial-NoDerivatives 4.0 International License (CC BYNC-ND) (http://www.karger.com/Services/OpenAccessLicense). Usage and distribution for commercial purposes as well as any distribution of modified material requires written permission. 
In most of the cases, onychomycosis is caused by anthropophylic dermatophytes of the Trichophyton species. Particularly, Trichophyton rubrum is the most common cause, followed by Trichophyton interdigitale. Scopulariopsis brevicaulis, Fusarium spp., and Aspergillus spp. are the most common non-dermatophyte molds isolated in onychomycosis, usually in the toenails. Other molds that have been isolated include Acremonium spp., Alternaria spp., Scytalidium spp., and other less frequent species. Yeasts represent the last common cause of nail fungal infection, and Candida albicans and Candida parapsilosis are the two most common isolates. Candida onychomycosis is seen in the hands of immunodepressed and diabetic patients [2] and in patients under chronic steroid therapy.

Onychomycosis can be associated with local pain, paresthesia, reduced quality of life, and impaired social interactions and daily activities [3]. Both the toenails and fingernails can be affected, with the toenails being more commonly involved, and it is commonly associated with a history of tinea pedis or hyperhidrosis [4]. Clinically, there are different clinical types of onychomycosis, depending on the modality of nail invasion: distal subungual onychomycosis (DSO), the most common type, white superficial onychomycosis, proximal subungual onychomycosis, or total onychomycosis.

The clinical signs of DSO include white or yellow nail discoloration, nail plate thickening with subungual hyperkeratosis and onycholysis. The diagnosis of onychomycosis can be suspected on clinical features alone but laboratory isolation of the fungus through direct microscopy with potassium hydroxide $(\mathrm{KOH})$ and culture examination still remains the gold standard.

Treatment is chosen depending on the modality of nail invasion, fungus species, and the number of affected nails. The difficulty in treating onychomycosis results from the deep-seated nature of the infection within the nail unit and the difficulty of drugs to effectively reach all sites. In case of DSO involving more than $50 \%$ of the nail and more than 3 nails, systemic oral therapy with terbinafine, itraconazole, or fluconazole represents the first choice of treatment. On the contrary, if the nail invasion is restricted to less than $50 \%$ of the nail and less than 3 nails are involved, the treatment is based on topical application of antifungals, conveyed in cream, gel, or nail lacquers [2]. Topical therapy is generally preferred both by patients and physicians because it is associated with lower risk of systemic side effects and drug interactions, avoiding laboratory monitoring [5]. However, the nail has a slow growth rate and its composition makes it a formidable barrier to the permeation and diffusion of drugs, requiring the right vehicle and a long duration of therapy, usually 6-8 months or longer, ideally until a healthy nail has regrown [6]. The goal of onychomycosis therapy is both to eliminate the infecting fungal organism and to restore the normal appearance of the nail (mycological and clinical cure, respectively). However, we must not forget that clinical cure does not always follow mycological cure, because toenails often show traumatic alterations or dystrophies that are not reversed by antifungal therapy.

The aim of our study was to evaluate the efficacy and tolerability of a new topical antifungal containing vitamin $\mathrm{E}$ and essential oil of lime, oregano, and tea tree. This combination of essential oils, with low percentage vehiculated in tocopheryl acetate, resulted in the most active agents against dermatophytes and molds in in vitro tests.

\section{Materials and Methods}

We enrolled 20 patients ( 13 males and 7 females, mean age: 45 years) with mild-moderate onychomycosis due to both dermatophytes, T. rubrum (14/20) and T. interdigitalis (3/20), and nondermatophyte molds, Fusarium sp. (2/20) and Scopulariopsis bevicaulis $(1 / 20)$. For all patients, the diagnosis was established through clinical examination, direct microscopy and/or culture examination. The viscous oily product, consisting of only 4 ingredients in a patented formulation, was applied once daily on the periungual tissues and the nail plate for 6 months, but follow-up without therapy continued for another 6 months in order to evaluate the product's effect maintenance. Periodic evaluation of treatment efficacy was performed by standardized photography and mycology of the target nail at baseline (T0), after 3 months (T1) and 6 months (T2) of therapy, and after 6 months of follow-up (T3). Treatment efficacy and tolerability were also subjectively evaluated by patients, through a specific questionnaire exploring efficacy, tolerability, easiness of application, and pleasantness of the oil. None of the patients was receiving any other systemic and/or topical treatment.

\section{Results}

Fourteen of the 20 enrolled patients concluded the study. Interestingly, among the 6 patients who dropped out of the study, 4 stopped treatment before the scheduled time because they achieved a complete cure of the affected nail ( 2 patients at $\mathrm{T} 1$ and 2 patients at $\mathrm{T} 2$ ) and did not want to come to visit.

Patients were affected by mild-moderate onychomycosis due to T. rubrum (14/20), T. interdigitalis $(3 / 20)$, Fusarium sp. (2/20), and Scopulariopsis bevicaulis (1/20), proved by mycology. After 3 months of therapy (T1), 17 of the 20 enrolled patients were evaluated, because 2 of 
them reported a complete resolution and 1 dropped out of the study for personal reasons. Of the examined patients, 3 patients were completely cured (17.6\%) (Fig. 1), $13(76.4 \%)$ showed a considerable improvement, and 1 remained stable (5.8\%).

After 6 months of therapy (T2), 14 of the 17 patients examined in $\mathrm{T} 1$ came back to the follow-up visit (among the 3 dropouts, 2 reported a complete resolution and 1 dropped out for personal reasons). Seven of the 14 patients (50\%) showed a complete cure of onychomycosis (Fig. 2), while 6 patients showed a significant improvement (42.8\%) (Fig. 3) and 1 remained stable (7.1\%).

Once the treatment was suspended, patients were asked to come back to the follow-up visit after 6 months (T3) in order to evaluate long-term efficacy: 11 patients achieved a complete resolution (78.5\%) (Fig 4), 2 patients showed an important improvement, and 1 remained stable. Overall, 15 patients were cured by this new treatment, 2 showed a significant improvement, 1 remained stable, and 2 patients dropped out.

All patient found the treatment easy to apply with a pleasant texture and the product did not leave sticky residues or any sensation on the nails. No treatment-related side effects were recorded in any patient. All the 17 patients who were able to fill out the patients' questionnaire were very satisfied by the treatment and would buy it if marketed. It is interesting to note that 11 patients stated to significantly improve after 3 months of therapy.

\section{Discussion}

Onychomycosis is a very common fungal infection, which needs a targeted treatment. Therapy requires several months, as the nail grows very slowly, especially in the elderly. Drug choice relies on the type and severity of onychomycosis and the associated comorbidities.

The most common type of onychomycosis is DSO and lateral subungual onychomycosis, which is characterized by a fungal invasion starting from the lateral or distal sites of the nail plate. The affected nails usually show subungual hyperkeratosis, onycholysis, and white or yellow discoloration. DSO usually affects one or both of the great toenails and is also usually associated with tinea pedis.

The choice of treatment for onychomycosis is often a challenge for the dermatologist and is influenced by several factors, such as the number of toes/fingers involved, severity of the disease, and presence of associated systemic diseases. Topical treatments are often the first choice in mild-moderate cases due to their lower risk of systemic side effects and drug interactions, but their effectiveness is limited to the presence of hyperkeratosis, which interferes with their absorption at the level of the nail plate. Penetration of a topical antifungal through the nail plate requires a vehicle that is specifically formulated for transungual delivery.

The topical product utilized in this study for treating mild to moderate DSO is very innovative in the formula-

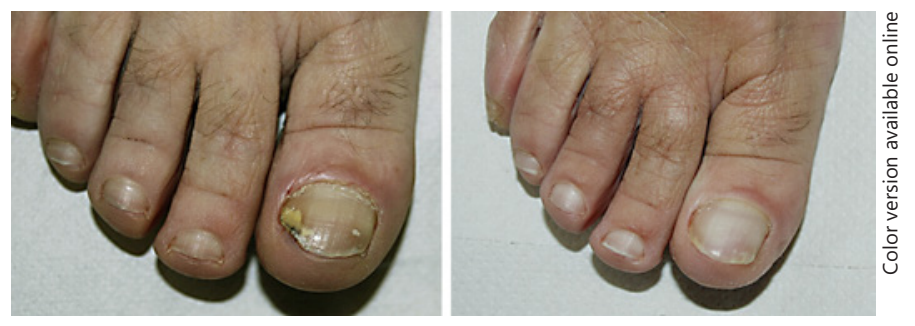

Fig. 1. A patient affected by onychomycosi completely cured after 3 months of therapy.
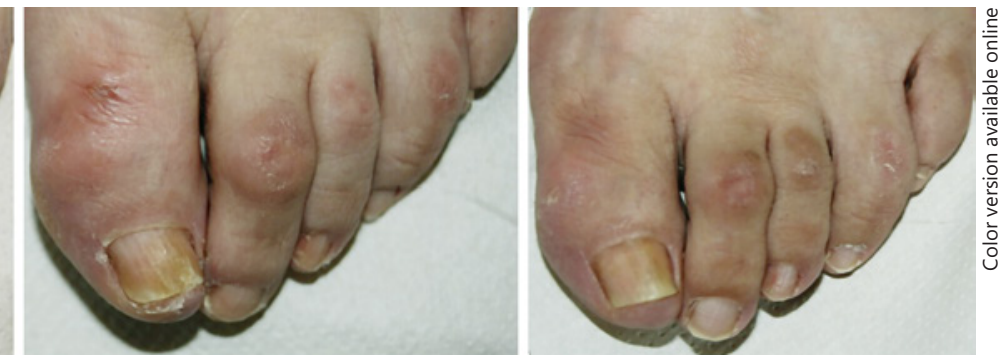

Fig. 2. A patient affected by onychomycosis completely cured after 6 months of therapy. 
tion, thanks to a result of to the combination of natural components. Vitamin E (tocopherol tocopheryl acetate) has a high antioxidant activity in its pure state and can accelerate cellular proliferation of fibroblasts and epithelial cells, favoring tissue growth [7]: for this reason, it has been widely used in dermatology for years to improve
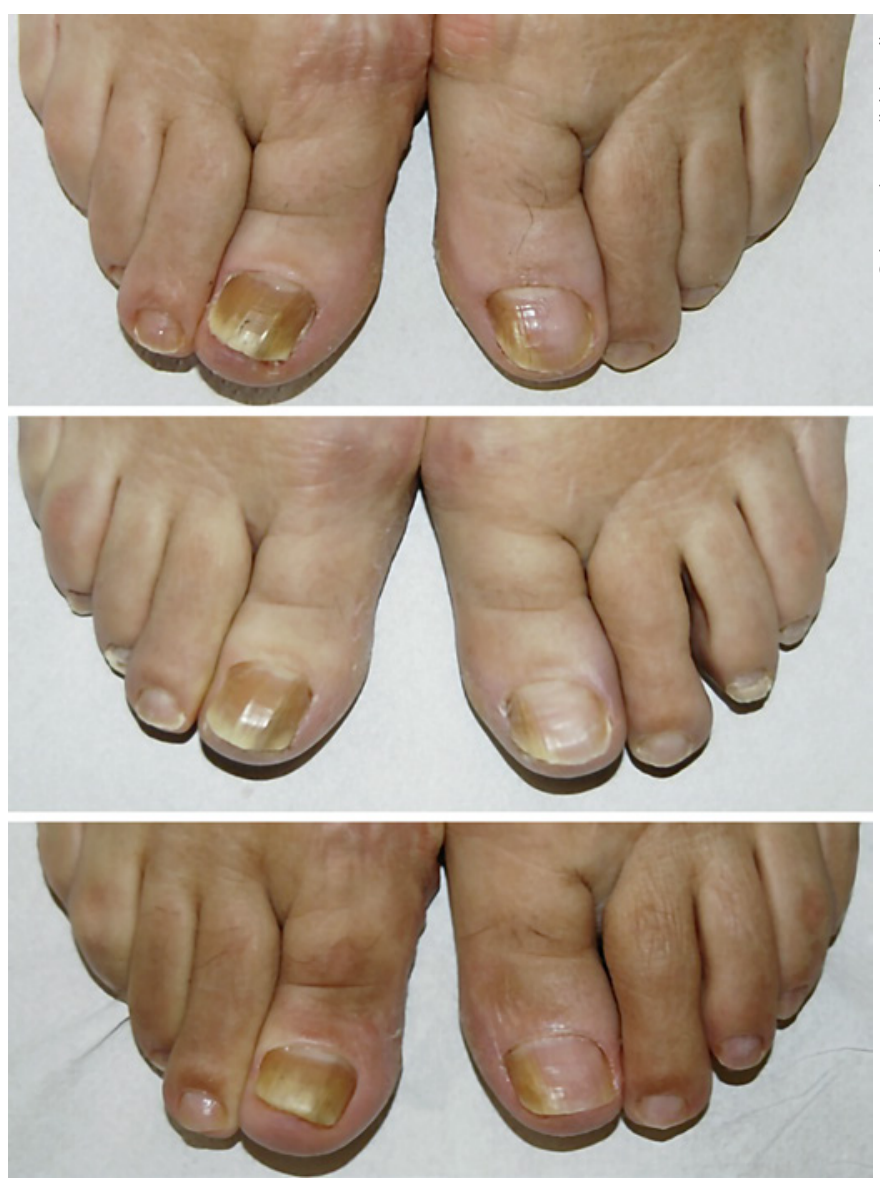

Fig. 3. A patient affected by onychomycosis with a significant improvement after 6 months of therapy. tissue trophism. In nail diseases, oral tocopherol acetate is one of the few effective treatments for yellow nail syndrome due to its antioxidant effect and because it increases the speed of nail growth [8]. Recent studies showed the high capacity of topical tocopherol tocopheryl acetate in conveying pharmacological molecules in tissues [9].

Tea tree oil is an essential oil obtained from Melaleuca alternifolia leaves, which, incorporated into topical formulations, has shown antibacterial, antifungal, and antiinflammatory properties $[10,11]$ with an exceptional skin penetration [12]. Oregano essential oil from Origanum vulgare leaves is able to inhibit the growth of various pathogenic bacteria and fungi [13-16]. Lime essential oil from Citrus aurantifolia peel has antimicrobial activity against bacteria and fungi $[13,17,18]$. In West Africa, lime is an essential ingredient in herbal medicine to treat various infectious diseases, burns, and gonorrhea because it is easily available and cheaper than the conventional drugs. Lime mesocarp helps in prevention of pimples due to its cleansing action on the skin [17].

The results obtained in our study indicate that this new topical antifungal containing vitamin $\mathrm{E}$ and essential oil of lime, oregano, and tea tree is an effective and safe option for topical therapy of onychomycosis and it can certainly represent one of the first choices of treatment in these patients. In addition, this topical formulation is well absorbed at the level of the nail plate, improving tropism of the nail and the periungual tissues, and makes the nail even more resistant to infections, thus reducing the risk of relapses.

The clinical appearance of the nail already improves from the first weeks of treatment, potentially contributing to a better compliance adherence in long-term therapy. In conclusion, our study showed that this new topical antifungal is not only able to obtain excellent therapeutic results but is also capable of maintaining such results over time.
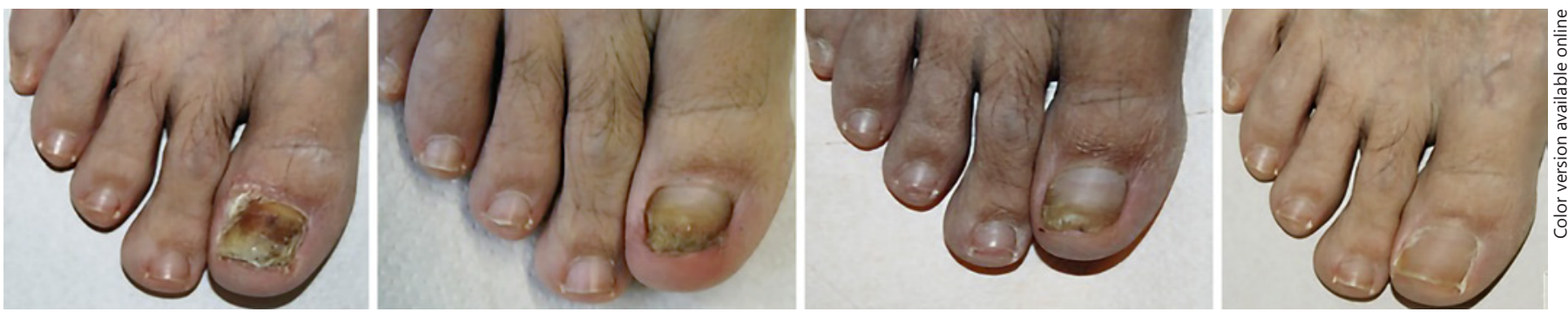

Fig. 4. A patient affected by onychomycosis completely cured after 6 months of follow-up.

Vitamin E and Essential Oils for

Onychomycosis
Skin Appendage Disord 2020;6:14-18 DOI: $10.1159 / 000503305$ 


\section{Statement of Ethics}

Subjects have given their informed consent and the study protocol has been approved by the institute's committee on human research.

\section{Disclosure Statement}

No conflicts of interest are declared.

\section{Funding Sources}

None.

\section{References}

1 Piraccini BM, Alessandrini A. Onychomycosis: A Review. J Fungi (Basel). 2015 Mar;1(1): $30-43$.

2 Gupta AK, Mays RR, Versteeg SG, Piraccini BM, Takwale A, Shemer A, et al. Global perspectives for the management of onychomycosis. Int J Dermatol. 2019 Oct;58(10):111829.

3 Gupta AK, Stec N. Recent advances in therapies for onychomycosis and its management. F1000Res. 2019 Jun 25;8. pii: F1000 Faculty Rev-968.

4 Lipner SR, Scher RK. Onychomycosis: clinical overview and diagnosis. J Am Acad Dermatol. 2019 Apr;80(4):835-51.

5 Lipner SR, Scher RK. Onychomycosis: treatment and prevention of recurrence. $J$ Am Acad Dermatol. 2019 Apr;80(4):853-67.

6 Angelo T, Borgheti-Cardoso LN, Gelfuso GM, Taveira SF, Gratieri T. Chemical and physical strategies in onychomycosis topical treatment: A review. Med Mycol. 2017 Jul; 55(5):461-75.

7 Caddeo C, Manca ML, Peris JE, Usach I, DiezSales O, Matos M, et al. Tocopherol-loaded transfersomes: in vitro antioxidant activity and efficacy in skin regeneration. Int J Pharm. 2018 Nov;551(1-2):34-41.

8 Vignes S, Baran R. Yellow nail syndrome: a review [Review]. Orphanet J Rare Dis. 2017 Feb;12(1):42.
9 Ahmed OA, El-Say KM, Aljaeid BM, BadrEldin SM, Ahmed TA. Optimized vinpocetine-loaded vitamin E D- $\alpha$-tocopherol polyethylene glycol 1000 succinate-alpha lipoic acid micelles as a potential transdermal drug delivery system: in vitro and ex vivo studies. Int J Nanomedicine. 2018 Dec;14:3343.

10 Flores FC, de Lima JA, Ribeiro RF, Alves SH, Rolim CM, Beck RC, et al. Antifungal activity of nanocapsule suspensions containing tea tree oil on the growth of Trichophyton rubrum. Mycopathologia. 2013 Apr;175(3-4): 281-6.

11 Buck DS, Nidorf DM, Addino JG. Comparison of two topical preparations for the treatment of onychomycosis: melaleuca alternifolia (tea tree) oil and clotrimazole. J Fam Pract. 1994 Jun;38(6):601-5.

12 Syed TA, Qureshi ZA, Ali SM, Ahmad S, Ahmad SA. Treatment of toenail onychomycosis with $2 \%$ butenafine and $5 \%$ Melaleuca alternifolia (tea tree) oil in cream. Trop Med Int Health. 1999 Apr;4(4):284-7.

13 Hammer KA, Carson CF, Riley TV. Antimicrobial activity of essential oils and other plant extracts. J Appl Microbiol. 1999 Jun; 86(6):985-90.
14 Chaftar N, Girardot M, Labanowski J, Ghrairi T, Hani K, Fre're Frère J, et al. Comparative evaluation of the antimicrobial activity of 19 essential oils. Advs Exp. Medicine, Biology Advances in Microbiology, Infectious Diseases and Public Health; 2015. https://doi. org/10.1007/5584_2015_5011.

15 Souza EL, Stamford TL, Lima EO, Trajano VN. Effectiveness of Origanum vulgare L. essential oil to inhibit the growth of food spoiling yeasts. Food Control. 2007;18(5):409-13.

16 Vinciguerra V, Rojas F, Tedesco V, Giusiano G, Angiolella L. Chemical characterization and antifungal activity of Origanum vulgare, Thymus vulgaris essential oils and carvacrol against Malassezia furfur. Nat Prod Res. 2018 May; $1-5$.

17 Aibinu I, Adenipekun T, Adelowotan T, Ogunsanya T, Odugbemi T. Evaluation of the antimicrobial properties of different parts of Citrus aurantifolia (lime fruit) as used locally. Afr J Tradit Complement Altern Med. 2006 Nov;4(2):185-90.

18 Ezikanyi DN, Nnamani CV, Ihejirika JN. Qualitative Phytochemical Analysis and in Vitro Fungal Activities of Citrus aurantifolia L. and Carica papaya L. Leaves Extracts on Pathogenic Fungi. Journal of Pharmacy and Biological Sciences. 2016;11:68-72. 